\title{
Rituximab Improves Overall Survival in Patients Treated with CODOX-M/IVAC for Burkitt Lymphoma (BL) and B-Cell Lymphoma, Unclassifiable, with Features Intermediate between Diffuse Large B-Cell Lymphoma and BL (BCL-U): A Single Center Experience and Review of the Literature
}

\author{
A. Prica1, A. Pratzer ${ }^{2}$, M. Cheung1, K. Thompson ${ }^{3}$, E. Piliotis' ${ }^{1}$, N. L. Berinstein', K. Imrie1, \\ R. Pradhan", A. Vyas', Z. Ghorab ${ }^{4}$, D. Good4 ${ }^{4}$, L. Zhang1, R. Buckstein ${ }^{*}$ \\ ${ }^{1}$ Hematology/Oncology, Odette Cancer Centre, Sunnybrook Health Sciences Centre, Toronto, Canada \\ ${ }^{2}$ Feinberg School of Medicine, Northwestern University, Chicago, USA \\ ${ }^{3}$ Schulich School of Medicine and Dentistry, University of Western Ontario, London, Canada \\ ${ }^{4}$ Department of Pharmacy, Sunnybrook Health Sciences Centre, Toronto, Canada \\ Email: rena.buckstein@sunnybrook.ca
}

Received 24 September 2014; revised 28 October 2014; accepted 10 November 2014

Copyright (C) 2015 by authors and Scientific Research Publishing Inc.

This work is licensed under the Creative Commons Attribution International License (CC BY).

http://creativecommons.org/licenses/by/4.0/

(c) (i)

Open Access

\section{Abstract}

Background: Dose-modified (dm) CODOX-M/IVAC is commonly used for Burkitt lymphoma (BL) and B-cell lymphoma, unclassifiable, with features intermediate between diffuse large B-cell lymphoma (DLBCL) and BL (BCL-U; previously Burkitt-like lymphoma-BLL). Methods: We evaluated the clinical characteristics, outcomes and prognostic markers in patients treated with dmCODOXM/IVAC+/- rituximab (R) at a single academic center. Results: 31 patients with BL $(n=21)$ or BCL-U $(n=10)$ were included. The median age was 45 , and $90 \%$ were high risk. The 2 -year overall survival (OS) and progression-free survival (PFS) were $69 \%$ and $62 \%$, respectively, with no differences between BL and BCL-U. By multivariable analysis, rituximab use was significantly associated with improved OS (Hazard Ratio $0.15 ; 95 \%$ CI 0.04 - 0.56) while elevated LDH (Hazard Ratio $2.84,95 \%$ CI 1.52 - 5.29) and rituximab use (Hazard Ratio 0.054, 95\% CI 0.01 - 0.32 ) predicted PFS. Conclusion: DmCODOX-M/IVAC+/- R chemotherapy provides equivalent survival for both BL and

${ }^{*}$ Corresponding author.

How to cite this paper: Prica, A., et al. (2015) Rituximab Improves Overall Survival in Patients Treated with CODOX-M/IVAC for Burkitt Lymphoma (BL) and B-Cell Lymphoma, Unclassifiable, with Features Intermediate between Diffuse Large B-Cell Lymphoma and BL (BCL-U): A Single Center Experience and Review of the Literature. Journal of Cancer Therapy, 6, 1-11. http://dx.doi.org/10.4236/jct.2015.61001 
BCL-U patients. The addition of rituximab improves overall and progression free survival and is recommended.

\author{
Keywords
}

\author{
Burkitt Lymphoma, Burkitt-Like Lymphoma, CODOX-M/IVAC, MYC, Rituximab
}

\title{
1. Introduction
}

Sporadic Burkitt lymphoma (BL) is a rare and highly aggressive B-cell non-Hodgkin's lymphoma (NHL) that often presents at extranodal sites or as an acute leukemia. It is characterized by a growth fraction approaching $100 \%$ and a characteristic immunohistochemical profile (CD20+, CD10+, BCL6+, BCL2-), and it accounts for $1 \%-2 \%$ of NHL in adults [1]. In addition to a characteristic histopathology and immunohistochemistry, most cases have MYC translocations at band 8q24 to the IG heavy chain region 14q32 or less commonly, at the lambda 22q11 or kappa 2p12 light chain loci [2].

B-cell lymphoma, unclassifiable (BCL-U), with features intermediate between diffuse large B-cell lymphoma (DLBCL) and BL (World Health Organization (WHO) 2008; previously classified as Burkitt like lymphoma (BLL)) is a highly aggressive lymphoma with morphological and genetic features of both DLBCL and BL. Thirty-five to fifty percent of the cases have 8q24/MYC translocations although many of these cases have other translocations (non IG-MYC). Approximately 15\% - 30\% of the cases with MYC translocation also have a BCL2 translocation and are referred to as "double hit" lymphomas [1].

In recent years, many adult cases of BL and BCL-U have been treated with a rapidly cycling, high intensity pediatric regimen developed by Ian Magrath comprised of 2 alternating cycles of CODOX-M (hyperfractionated cyclophosphamide, doxorubicin, vincristine, high-dose methotrexate with folinic acid rescue, intrathecal cytarabine and methotrexate, and granulocyte colony-stimulating factor (G-CSF)) and IVAC (ifosfomide, mesna, cytarabine, etoposie, intrathecal methotrexate and G-CSF) [3] [4]. This rapidly cycling, high-intensity chemotherapy, inclusive of aggressive central nervous system (CNS) prophylaxis, has cured a high proportion of BL patients, but is toxic, with high rates of febrile neutropenia and neuropathy and a treatment related mortality (TRM) as high as $17 \%$ [5]. More recently, CODOX-M has been modified (dmCODOX-M) for adults, by reducing systemic methotrexate [5] [6], increasing doxorubicin dosing [6], consolidating cyclophosphamide dosing to 2 days [6], capping the vincristine dose to $2 \mathrm{mg}$ [5]-[7] and making further dose modifications in patients over the age of 65 [5]. The addition of rituximab to CODOX-M/IVAC is relatively new in both BL and BCL-U [8]-[11]. Because of an ambivalence regarding the treatment of BCL-U as BL or DLBCL, some groups are starting to add extra therapy, such as additional R-CODOX cycles after CODOX-M/IVAC protocol completion, in order to maximize anthracycline and rituximab delivery [11]. Much of the existing literature surrounding BL and the CODOX-M/IVAC regimen has involved small sample sizes with a relatively young median age with mixed histologies (Table 3).

We undertook this retrospective study of patients treated at a large quaternary care cancer center with CODOX-M and IVAC in order to a) determine "real-world" toxicity, compliance and survival rates, b) examine the effects of BL versus intermediate histology diagnoses, and c) evaluate the impact of rituximab (R) use on clinical outcomes.

\section{Methods}

\subsection{Study Design}

Patients at Sunnybrook Health Sciences Centre in Toronto, Canada were retrospectively identified from January 2006-December 2012. In total, 31 HIV negative patients were identified who had been diagnosed as having either Burkitt lymphoma or atypical Burkitt/Burkitt-like lymphoma (WHO Classification 2001) [12], or B-cell lymphoma, unclassifiable, with features intermediate between diffuse large B-cell lymphoma and BL (BCL-U; WHO Classification 2008) [1] and who had received the CODOX-M/IVAC regimen, +/- R within 2 months of their diagnosis. Patients who had received CHOP-like chemotherapy (cyclophosphamide, vincristine, doxorubi- 
cin and prednisone) +/- R initially for an equivocal diagnosis were included if they were eventually reclassified as BL or BCL-U and received at least one cycle of dmCODOX-M and/or IVAC [7]. Criteria needed for diagnosis including morphology, proliferation index, immunophenotype, and cytogenetics in addition to clinical outcomes were obtained from inpatient and outpatient medical records.

Tumor samples were centrally reviewed at Sunnybrook Health Sciences Centre by hematopathologists who specialize in lymphoproliferative disorders. BL was defined if the histology was concordant with the WHO definition [1]. Lymphomas with features of intermediate histology and MYC +/- BCL-2 expression were called BLL prior to 2008 and more recently B-cell lymphoma, unclassifiable, with features intermediate between DLBCL and BL (BCL-U), which were considered equivalent between the two eras. BCL-2 expression was defined as positive by immunohistochemistry if $\geq 30 \%$ of cells were involved. Fluorescence in situ hybridization (FISH) was performed for MYC and BCL-2 rearrangements. These cases were left classified in aggregate as BCL-U for this report. Double hit lymphomas were defined by the detection of a concurrent $t(14 ; 18)$ and MYC translocation.

Patients at Sunnybrook Health Sciences Centre were generally treated uniformly according to an internal protocol, although adjustments according to the individual patient context was allowed and left to the discretion of treating physicians. The dmCODOX-M/IVAC $+/-\mathrm{R}$ was given to all Burkitt lymphoma patients and BCL-U patients, as classified histologically by the hematopathology central review. Patients were classified as low risk (LR) if they had a normal lactate dehydrogenase (LDH), Ann Arbor stage 1 - 2 disease, a performance status of $0-1$ and no tumor mass $>10 \mathrm{~cm}$ [7]. Low risk (LR) patients received 3 cycles of dmCODOX-M as described by Mead et al. [7]. All other patients were classified as high risk (HR) and received 2 cycles of dmCODOX-M (or 1 cycle each of CHOP-R and dmCODOX-M, respectively) and 2 cycles of dmIVAC [7]. In 2012, our approach to BCL-U was altered, by adding 1 - 4 cycles of R-CHOP after the dmCODOX-M/IVAC protocol completion, to maximize anthracycline and rituximab delivery.

\subsection{Evaluation of Response}

The primary outcomes of interest were 2-year overall (OS) and progression-free survival (PFS). PFS and OS were both calculated from date of diagnosis. Our secondary outcomes included complete response (CR), partial response (PR) and overall response rates (ORR) using the Cheson criteria [13]. Patients were considered evaluable if they completed the planned cycles of therapy and had CT scans afterwards to evaluate response, or they had progressive disease during primary therapy. Additional secondary outcomes included TRM, rates of CNS relapse, first chemotherapy other than CODOX-M, rituximab use and addition of R-CHOP after the last IVAC. We also assessed compliance with the protocol and key toxicities.

\subsection{Statistical Considerations}

Data are presented descriptively in all patients and by BL or BCL-U. The Kaplan-Meier survival curve and 95\% confidence interval (CI) was used to describe OS and PFS in all patients. Subgroup survival analyses were also conducted using a log-rank test comparing rituximab (yes/no) use, BL versus BCL-U, commencing with CHOP$\mathrm{R}$ versus CODOX-M, R-CHOP after (+/-) and CNS involvement (+/-) at diagnosis. Univariable and multivariable Cox proportional hazard models of OS and PFS were constructed. Two-sided $p$-value $<0.05$ was considered as statistical significant. All analyses were conducted by Statistical Analysis Software package (SAS version 9.3 for Windows).

\subsection{Ethics}

This study was approved by the research ethics board at Sunnybrook Health Sciences Center, in accordance with the general ethical principles outlined in the Declaration of Helsinki and the Tri-Council Policy Statement: Ethical Conduct for Research Involving Humans (TCPS).

\section{Results}

\subsection{Patient Characteristics}

Thirty-one patients diagnosed between Jan 2006 and December 2012 were identified and included in this study. 
Twenty-one and ten were considered to be BL and BCL-U patients, respectively. The median age was 45 years (range 19 - 74), and 80\% of the patients were male. Twenty-eight patients (90\%) were HR, and 21 patients (68\%) had Ann Arbor stage > 2. Three (10\%) had baseline CNS involvement. FISH was available for 30 out of 31 patients. MYC was rearranged in 29 of the 30 cases with FISH available. Additionally, 8 (26\%) patients were BCL2+ by immunohistochemistry (IHC). Three were also positive for the $t(14 ; 18)$ (double-hit lymphomas), and one was unknown. Patient characteristics are summarized in Table 1.

\subsection{Treatment}

Because of the diagnostic uncertainty (pending MYC status), 10 (32\%) began treatment with chemotherapy other than dmCODOX-M/IVAC. For 9 of 10 patients, initial chemotherapy consisted of 1 - 2 cycles of CHOP-R, with the addition of high-dose systemic methotrexate (HDMTX $3 \mathrm{~g} / \mathrm{m}^{2}$ ) for CNS prophylaxis on day 10 (CHOMP-B) [14]. One patient had a cycle of carboplatin and taxol for suspected ovarian carcinoma, which was then proved to be BL. In 3 patients, this first cycle effectively substitututed for cycle 1 of dmCODOX-M when the diagnosis was revised to BL or BCL-U. Six of these patients subsequently completed 2 dmCODOX-M and 2 dmIVAC cycles, while four only completed dmCODOX-M $\times 1$ with 2 dmIVAC cycles.

In May 2008, we adopted the practice of adding R $375 \mathrm{mg} / \mathrm{m}^{2}$ to dmCODOX-M/IVAC in non-HIV BL patients due to emerging data from other centers [15]. Nineteen (61\%) patients received $\mathrm{R}$ with treatment (day 1 or 2 of dmCODOX-M and dmIVAC), and 10 (32\%) patients received R as part of a preceding CHOP-R cycle. In total, 22 (71\%) patients received at least one infusion of rituximab at some point during first line treatment with a median of 3 infusions (range 1 - 7). There were no significant differences in baseline characteristics between patients who received rituximab and those that did not (data not shown). All patients received intrathecal chemotherapy per protocol [7].

In January 2012, we added additional cycles of CHOP-R (at physician's discretion) after completion of dmCODOX-M/IVAC in patients with BCL-U. Five of 10 BCL-U patients (50\%) received a median of 2 additional R-CHOP cycles (range 1 - 4).

Table 1. Patient demographics.

\begin{tabular}{|c|c|c|c|}
\hline & $\operatorname{ALL}(n=31)$ & $\operatorname{BL}(n=21)$ & BCL-U $(n=10)$ \\
\hline $\begin{array}{l}\text { Age at diagnosis } \\
\text { Median (range) }\end{array}$ & $45(19-74)$ & $50(19-74)$ & $33(27-65)$ \\
\hline c-Myc translocation (n) & 29 & 19 & 10 \\
\hline $\begin{array}{c}\text { BCL-2 + (n) } \\
\text { Bcl2 translocation (n) } \\
\text { Ki67 } \% \text { ) }\end{array}$ & $\begin{array}{l}8 \\
3\end{array}$ & $\begin{array}{l}3 \\
0\end{array}$ & $\begin{array}{l}5 \\
3\end{array}$ \\
\hline Median (range) & $99(40-100)$ & $100(40-100)$ & $95(90-100)$ \\
\hline $\begin{array}{c}\text { LDH } \\
\text { Median (range) }\end{array}$ & $519(136-10940)$ & $473(136-3934)$ & $520(187-10940)$ \\
\hline $\begin{array}{l}\text { Risk Level } \\
\text { HR (n) } \\
\text { LR (n) }\end{array}$ & $\begin{array}{c}28 \\
3\end{array}$ & $\begin{array}{c}19 \\
2\end{array}$ & $\begin{array}{l}9 \\
1\end{array}$ \\
\hline $\begin{array}{c}\text { Clinical Stage } \\
1 \\
2 \\
3 \\
4\end{array}$ & $\begin{array}{c}2 \\
8 \\
5 \\
16\end{array}$ & $\begin{array}{c}2 \\
5 \\
3 \\
11\end{array}$ & $\begin{array}{l}0 \\
3 \\
2 \\
5\end{array}$ \\
\hline CNS + at baseline (n) & 3 & 3 & 0 \\
\hline CHOP/CHOP-R/other first (n) & 10 & 6 & 4 \\
\hline $\begin{array}{c}\text { \# CODOX-M (median, range) } \\
\text { \# IVAC (median, range) }\end{array}$ & $\begin{array}{l}2(1-3) \\
2(0-2)\end{array}$ & $\begin{array}{l}2(1-3) \\
2(0-2)\end{array}$ & $\begin{array}{l}1(1-2) \\
2(0-2)\end{array}$ \\
\hline $\begin{array}{c}\text { Rituximab (n) } \\
\text { \# infusions (median, range) }\end{array}$ & $\begin{array}{c}22 \\
3(0-7)\end{array}$ & $\begin{array}{c}14 \\
2(0-4)\end{array}$ & $\begin{array}{c}8 \\
4(0-7)\end{array}$ \\
\hline Recommended Rx completed (n) & 22 & 14 & 8 \\
\hline
\end{tabular}

Legend: LDH: lactate dehydrogenase; HR: high risk; LR: low risk; Rx: treatment. 


\subsection{Chemotherapy Timing}

The median time from diagnosis to first chemotherapy was 13 days $(1-58)$ and from first chemo to first HDMTX 10.5 days ( 0 - 57). The median time between dmCODOX-M \#1/CHOP-R \#1 to dmIVAC \#1 was 28 days (17 - 75), from dmIVAC \#1 to dmCODOX-M \#2 was 22 days $(18$ - 32) and from dmCODOX-M \#2 to dmIVAC \#2 was 29 days (20-45). In the patients who received R-CHOP first vs. those who did not, the median times from diagnosis to the first chemotherapy were 9 days (range: 1 - 21) and 17 days (range: 0 - 58) respectively ( $\mathrm{p}=0.29)$.

\subsection{Toxicities}

Grades 3 - 4 neutropenia and thrombocytopenia were universal, despite the majority of patients receiving GCSF support (87\%). Twenty-four patients (77\%) experienced at least one episode of febrile neutropenia post dmCODOX-M and 12 patients (39\%) post dmIVAC, and 12 (39\%) experienced 1+ episodes of grades 2 - 3 mucositis. Eight (26\%) patients were admitted to the intensive care unit (ICU) for septic shock. There were five treatment-related deaths (TRM 16\%), all in HR patients due to sepsis.

\subsection{CNS Involvement}

Three patients (10\%) had CNS involvement at baseline. Two of the patients have died, 1 from relapsed disease and 1 from TRM. One patient remains alive in complete remission.

\subsection{Response and Survival}

Twenty-two patients (71\%) completed recommended therapy and 9 did not for the following reasons: primary progressive disease (PPD) $(n=3)$, TRM $(n=5)$ and toxicity $(n=1)$. Of 27 with evaluable disease, there were 20 with CR, 1 with PR and 6 with PPD. With a median time to death or last follow up of 15 months, 10 patients (32\%) died at a median time of 3.7 months (1.0 - 24.9). The causes of death were disease-related in 4, TRM in 5 and from unknown causes in 1 . Of 21 patients who are alive, one patient with a "double-hit" lymphoma experienced disease relapse at 2.7 years (with a DLBCL lacking the MYC and BCL2 gene translocations) and has undergone autologous stem cell transplantation in CR2. All three LR patients are alive in ongoing CR. Response to treatment by histology is summarized in Table 2 .

Two-year OS and PFS were 69\% (95\% CI 53\% - 89\%) and 68\% (95\% CI 53\% - 86\%), respectively and are summarized in Figure 1. Patients who received rituximab had superior OS (2-yr OS 86\% vs. 33\%, $p=0.0013$; Figure 2) compared with those that did not, regardless of whether it was received with CHOP and/or dmCODOX-M/IVAC. There was no difference in OS between BL and BCL-U (2-yr OS 64\% vs. 78\%, $p=0.37$; Figure 3). When BCL-U was separated by whether R-CHOP was given, there were still no differences in OS be-

Table 2. Clinical outcomes.

\begin{tabular}{|c|c|c|c|}
\hline & $\operatorname{ALL}(n=31)$ & $\mathrm{BL}(n=21)$ & BCL-U $(n=10)$ \\
\hline \multicolumn{4}{|l|}{ Remission status } \\
\hline $\mathrm{CR}(n)$ & 20 & 14 & 6 \\
\hline $\mathrm{PR}(n)$ & 1 & 1 & 0 \\
\hline $\operatorname{PPD}(n)$ & 6 & 4 & 2 \\
\hline \multicolumn{4}{|l|}{ Vital status } \\
\hline Alive $(n)$ & 21 & 13 & 8 \\
\hline \multicolumn{4}{|l|}{ Cause of death } \\
\hline TRM (n) & 5 & 4 & 1 \\
\hline NHL $(n)$ & 1 & 1 & 0 \\
\hline $\operatorname{PPD}(n)$ & 3 & 2 & 1 \\
\hline Unknown $(n)$ & 1 & 1 & 0 \\
\hline \multicolumn{4}{|l|}{ Relapse } \\
\hline CNS relapse $(n)$ & 2 & 1 & 1 \\
\hline Systemic relapse $(n)$ & 2 & 1 & 1 \\
\hline
\end{tabular}

Legend: CR: complete response; PR: partial response; PPD: primary progressive disease; TRM: treatment-related mortality; NHL: non Hodgkin’s lymphoma; BL: Burkitt lymphoma; BLL: Burkitt-like lymphoma. 


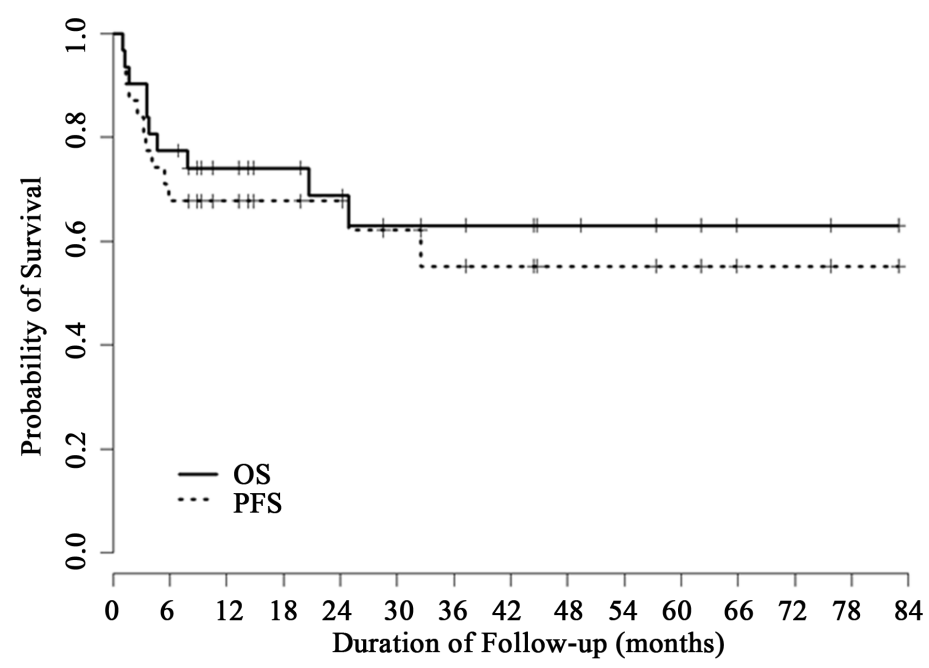

Figure 1. Probability of overall survival (OS) and progression-free survival (PFS) in all patients.
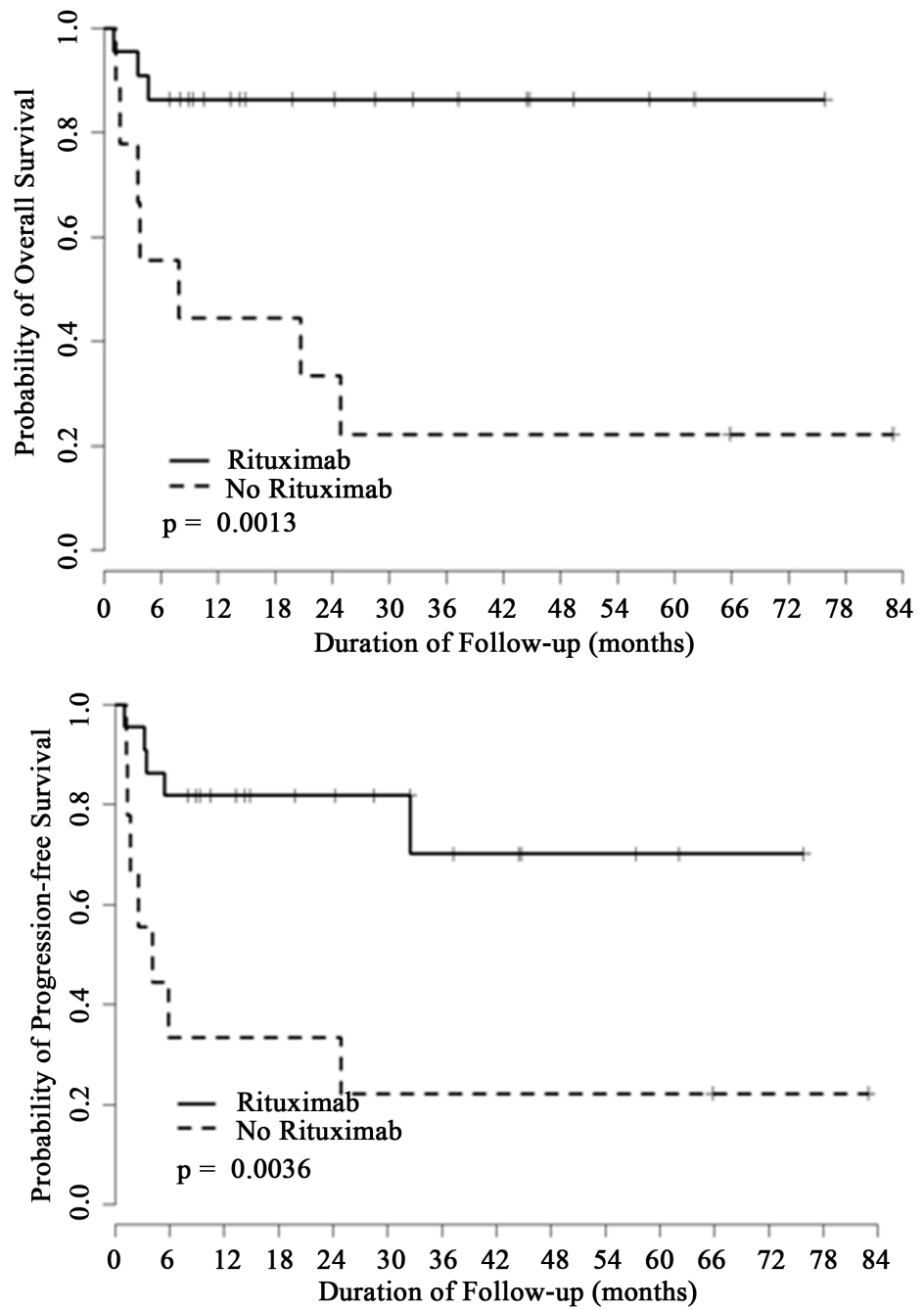

Figure 2. Probability of OS and PFS in patients who received rituximab vs. those that did not. 


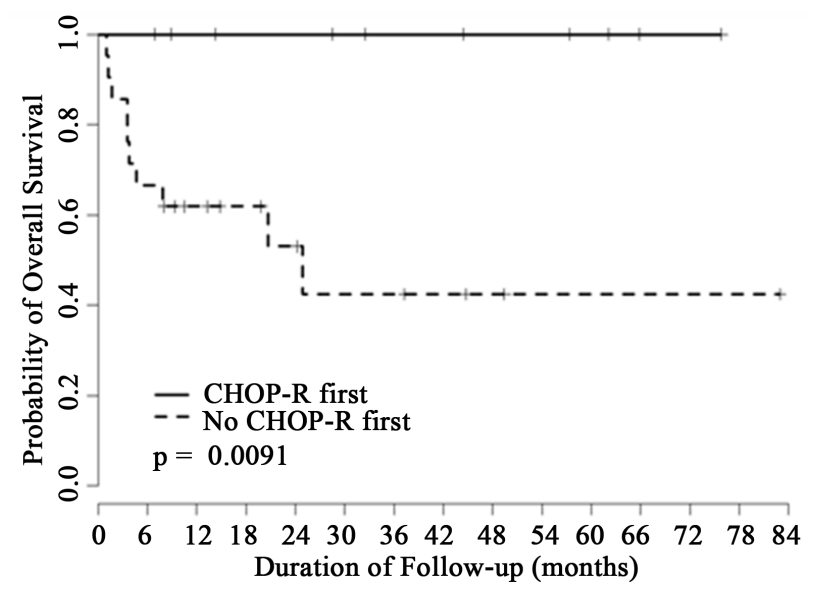

(a)

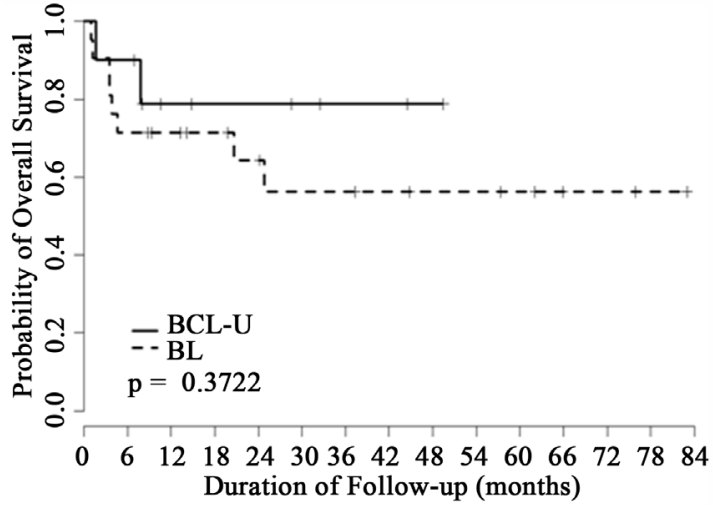

(b)

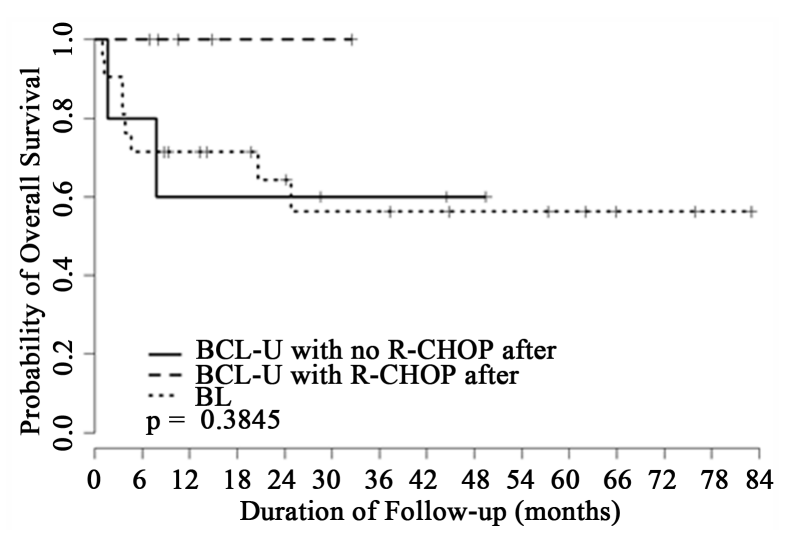

(c)

Figure 3. (a) Probability of OS in patients who received R-CHOP first vs. those that did not; (b) Probability of OS in BL vs. BCL-U patients, with BCL-U patients further subcategorized by extra R-CHOP after (c).

tween BL, BCL-U (“no R-CHOP after”) and BCL-U ("R-CHOP after”) (2-yr OS 64\% vs. 60\% vs. 100\%, p = 0.38; Figure 3). One of the double hit lymphoma patients received R-CHOP after and two did not.

Interestingly, initiating chemotherapy with CHOP-R versus dmCODOX-M was associated with an OS benefit (2-yr OS 100\% vs. 53\%, $p=0.009$; Figure 3), despite no observed differences in OS attributable to the variable ‘time from diagnosis to first chemotherapy $>$ or $<10$ days' $(p=0.96)$. Similarly, starting with CHOP-R versus dmCODOX-M $(p=0.02)$ and any rituximab use $(p=0.0036)$ demonstrated statistically different progressionfree survival curves (not shown).

\subsection{Multivariable Analysis}

By Cox proportional multivariate analysis, only rituximab use (Hazard Ratio 0.15, 95\% CI 0.04 - 0.56) was significant for OS, while elevated LDH (Hazard Ratio 2.84, 95\% CI 1.52 - 5.29) and rituximab use (Hazard Ratio 0.054, 95\% CI 0.01 - 0.32) predicted PFS.

\section{Discussion}

Sporadic Burkitt lymphoma is a rare, potentially curable B-cell lymphoma that predominantly occurs in younger adults [12] [16]. Published series to date of CODOX-M/IVAC for BL/BCL-U have been small, mainly singlecenter retrospective experiences with varied patient characteristics enrolled based on unreliable morphologic criteria and inconsistent detection of MYC rearrangement [7] [9]-[11] [17] (Table 3).

The MRC/NCRI LY10 trial [5] prospectively analyzed 110 aggressive B-cell lymphoma patients (58 BL, 70 DLBCL) who received dmCODOX-M +/- IVAC. The 2 year PFS/OS were 64\%/67\% for BL versus 55\%/59\% 
Table 3. Review of the literature.

\begin{tabular}{|c|c|c|c|c|c|c|c|c|c|c|c|c|}
\hline & $\mathbf{N}$ & $\begin{array}{l}\text { Median Age } \\
\text { (Range) }\end{array}$ & Regimen & Histologies & $\begin{array}{c}\mathrm{R} \\
(\%)\end{array}$ & $\begin{array}{l}\text { HIV } \\
\text { (\%) }\end{array}$ & $\begin{array}{l}\text { ORR } \\
(\%)\end{array}$ & $\begin{array}{l}\text { CR } \\
(\%)\end{array}$ & $\begin{array}{c}2 \text { Year } \\
\text { EFS (\%) }\end{array}$ & $\begin{array}{l}2 \text { Year } \\
\text { OS (\%) }\end{array}$ & $\begin{array}{c}\text { TRM } \\
(\%)\end{array}$ & $\begin{array}{c}\text { Completed } \\
\text { Therapy (\%) }\end{array}$ \\
\hline $\begin{array}{l}\text { Magrath [3] } \\
\text { (1996) }\end{array}$ & 41 & $\begin{array}{l}\text { Child = 12; } \\
\text { Adult = } 25 \\
\quad(3-59)\end{array}$ & $\begin{array}{l}\text { Protocol } \\
\text { 89-C-41 } \\
\text { (CODOX-M } \\
\text { IVAC) }\end{array}$ & SNCL & 0 & 0 & 100 & 95 & 92 & $95^{\mathrm{h}}$ & 0 & 93 \\
\hline $\begin{array}{l}\text { Mead [7] } \\
(2002)\end{array}$ & 52 & $\begin{array}{c}35 \\
(16-60)\end{array}$ & $\begin{array}{l}\text { Modified } \\
\text { 89-C-41 }\end{array}$ & $\begin{array}{l}\text { BL only, defined by } \\
\text { morphology and } \\
\text { Ki-67 of } 100 \%\end{array}$ & 0 & 0 & 87 & 77 & 65 & 73 & 12 & 79 \\
\hline $\begin{array}{l}\text { Lacasce [6] } \\
\text { (2004) }\end{array}$ & 14 & $\begin{array}{c}47 \\
(18-65)\end{array}$ & $\begin{array}{l}\text { Modified } \\
\text { 89-C-41 }\end{array}$ & $\begin{array}{l}\text { BL and BCL-U, } \\
\text { defined by morphology } \\
\text { and Ki- } 67 \geq 90 \%\end{array}$ & 0 & 0 & 86 & 86 & $64^{\mathrm{f}}$ & 71 & 0 & 100 \\
\hline $\begin{array}{l}\text { Mead [5] } \\
(2008)\end{array}$ & 110 & $\begin{array}{c}37 \\
(17-76)\end{array}$ & $\begin{array}{l}\text { Modified } \\
\text { 89-C-41 }\end{array}$ & $\begin{array}{l}\text { Aggressive B cell } \\
\text { lymphoma with } \\
\text { Ki67 fraction } \\
\text { approaching } 100 \%{ }^{\mathrm{i}}\end{array}$ & 0 & 0 & N/A & N/A & $64^{\mathrm{f}}$ & 67 & 17 & 72 \\
\hline $\begin{array}{l}\text { Maruyama [9] } \\
\text { (2010) }\end{array}$ & 15 & $\begin{array}{c}39 \\
(19-59)\end{array}$ & $\begin{array}{l}\text { Modified } \\
\text { 89-C-41 } \\
\text { R day } 1 \text { each } \\
\quad \text { cycle }\end{array}$ & $\begin{array}{l}\text { 4BL and } 11 \\
\text { intermediate } \\
\text { DLBCL/BL }\end{array}$ & 60 & 0 & 87 & 87 & 87 & 87 & 0 & 80 \\
\hline $\begin{array}{l}\text { Barnes [18] } \\
\text { (2011) }\end{array}$ & 80 & $\begin{array}{c}46 \\
(17-78)\end{array}$ & $\begin{array}{l}\text { Modified } \\
\text { 89-C-41 } \\
\text { R day } 1 \text { each } \\
\quad \text { cycle }\end{array}$ & $\begin{array}{l}\text { BL, defined by } \\
\text { REAL or WHO } \\
\text { criteria at diagnosis }\end{array}$ & 50 & 18 & 89 & 88 & $68^{g}$ & $71^{\mathrm{g}}$ & 6 & 87 \\
\hline $\begin{array}{c}\text { Mohamedbhai } \\
\text { [10] (2011) }\end{array}$ & 23 & $\begin{array}{c}\text { NR } \\
(19-65)\end{array}$ & $\begin{array}{l}\text { 89-C-41 } \\
\text { R days } 1,10 \\
\text { each cycle }\end{array}$ & $\begin{array}{l}\text { BCNHL with } \\
\text { proliferation } \\
\text { index }>95 \%\end{array}$ & 100 & 0 & 87 & 61 & N/A & $83^{\mathrm{h}}$ & 0 & 91 \\
\hline $\begin{array}{c}\text { Corazzelli [11] } \\
\text { (2012) }\end{array}$ & 30 & $\begin{array}{c}52 \\
(25-77)\end{array}$ & $\begin{array}{l}\text { Modified } \\
89-C-41 \\
\text { R days } 1 \text { of } \\
\text { each cycle }\end{array}$ & $\begin{array}{l}\text { BL and BCL-U, } \\
\text { defined by } \\
\text { morphology }\end{array}$ & 100 & 0 & 93 & 93 & $78^{\mathrm{j}}$ & $82^{j}$ & 0 & 80 \\
\hline $\begin{array}{l}\text { Wasterlid } \\
\text { [17] (2013) }\end{array}$ & 32 & 42 & $\begin{array}{l}\text { Modified } \\
\text { 89-C-41 } \\
\text { R days } 1 \text { of } \\
\text { each cycle }^{\text {e }}\end{array}$ & $\begin{array}{l}\text { BL only, defined } \\
\text { by morphology and } \\
\text { Ki-67 of }>95 \%\end{array}$ & 44 & 0 & NR & NR & NR & 69 & NR & NR \\
\hline $\begin{array}{l}\text { Present } \\
\text { study }\end{array}$ & 31 & $\begin{array}{c}45 \\
(20-74)\end{array}$ & $\begin{array}{l}\text { Modified } \\
89-C-41^{a}\end{array}$ & $\begin{array}{l}\text { BL and BCL-U, } \\
\text { defined by } \\
\text { morphology }\end{array}$ & 71 & 0 & 68 & 65 & $62^{f}$ & 69 & 16 & 71 \\
\hline
\end{tabular}

${ }^{\mathrm{a}}$ Omission of vincristine injection on day 15 of CODOX-M, vincristine capped at $2 \mathrm{mg} .{ }^{\mathrm{b}}(1)$ cyclophosphamide dose $800 \mathrm{mg} / \mathrm{m}^{2} / \mathrm{day}$ over 2 days (not 5), (2) vincristine capped at $2 \mathrm{mg}$, (3) IT cytarabine decreased to $50 \mathrm{mg}$ from $70 \mathrm{mg}$, (4) IV methotrexate reduced to $3 \mathrm{~g} / \mathrm{m} 2$ instead of $6.7 \mathrm{~g} / \mathrm{m}^{2}$ (4) doxorubicin increased to $50 \mathrm{mg}$ from $40 \mathrm{mg}$. 'Same as A but (1) MTX reduced to $3 \mathrm{~g} / \mathrm{m}^{2}$ from $6.7 \mathrm{~g} / \mathrm{m}^{2}$ (2) dose adjustments for age 65 y or older to IV MTX, ifosfamide and IV cytarabine. ${ }^{\mathrm{d}}$ Same as C but (1) mesna dose reduced to $300 \mathrm{mg} / \mathrm{m} 2$ from $360 \mathrm{mg} / \mathrm{m}^{2}$, (2) leukovorin reduced to 85.7 $\mathrm{mg} / \mathrm{m}^{2}$ from $192 \mathrm{mg} / \mathrm{m}^{2}$, (3) IT MTX increased to $15 \mathrm{mg}$ from $12 \mathrm{mg}$, (4) $20 \%$ dose reductions for age > 50. ${ }^{\text {e} A l l ~ B C L-U ~ p t s ~ r e c e i v e d ~} 2$ additional cycles of R-CODOX (with no IT, HDMTX or day 8 vincristine). ${ }^{\mathrm{f}} \mathrm{PFS}$ measurement. ${ }^{\mathrm{g}} @ 3$ years. ${ }^{\mathrm{h}}$ Percent patients alive at 2 years. ${ }^{\mathrm{B}} \mathrm{BL} / \mathrm{BCL}-\mathrm{U}$ and DLBCL included. BL characterized by presence of c-myc rearrangement as sole cytogenetic abnormality occurring in patients with germinal center phenotype with absence of BCL-2 expression and abnormal TP53 expression. ${ }^{j} @ 4$ years. Legend: TRM: treatment related mortality, CR: complete remission, OS: overall survival, EFS: event free survival, BCNHL: B cell non Hodgkin’s lymphoma, SNCL: small non-cleaved cell lymphoma, BCNHL: B-cell non Hodgkin's lymphoma.

for DLBCL ( $\mathrm{p}=\mathrm{NS})$. Negative prognostic markers were DLBCL, increasing age, CNS ivolvement and the presence of $\mathrm{t}(14 ; 18)$.

In our cohort of 31 patients with BL/BCL-U treated with dmCODOX-M/IVAC, we observed a completion rate of 71\%, CR in 65\%, PPD in 19\% and TRM in16\%. Two-year OS and PFS were 69\% and 68\%, respectively. These clinical results appear to be similar to previously published series including patients with and without $\mathrm{R}$ [5] [17] [18], but worse than series in which all patients received R (Table 3). Our TRM of 16\% is slightly higher than most, but comparable with Mead et al. [5], emphasizing the significant risks of this multi-agent cytotoxic chemotherapy regimen. As in other series, most TRM was infection-related and patients experienced high rates of grades 3 - 4 mucositis, sepsis and ICU admissions. As observed by others, late relapses were uncommon in 
our patients with only 1 patient relapsing at 2.7 years.

Despite modest numbers of rituximab infusions (median 3), we found that rituximab significantly improved overall and progression-free survival. This bolsters the findings of some groups [18] but contrasts with those of others [17]. More recently, Ribrag et al. (2012) reported in abstract form the results of a randomized controlled trial of COPADM (cyclophosphamide, vincristine, prednisolone, doxorubicin and methotrexate) $+/-$ R. The addition of R significantly improved 3-yr event-free survival (76\% vs. 64\%; $\mathrm{p}=0.046)$ and 3-yr OS (82\% vs. $71 \%$; $p=0.016$ ) [19]. The optimal number and schedule of $\mathrm{R}$ infusions added to Burkitt lymphoma chemotherapy is currently unknown.

Unfortunate delays in diagnosis are common with this disease in "real-world practice" for a number of reasons: 1 . morphologically this is a difficult diagnosis to make by general community pathologists, so $2^{\text {nd }}$ opinions at tertiary care centers are commonly sought, 2. MYC and BCL2 translocation resulting by FISH cytogenetics are not commonly done "in house" and often delayed by turnaround times ranging from 1 - 4 weeks. Thirty-two percent of our patients were initially misclassified as DLBCL and treated with chemotherapy other than CODOX-M for cycle \# 1. The literature would tell us that this is not an uncommon occurrence [5] [9], but highlights the importance of a faster detection of MYC status. There is new evidence validating the correlation between MYC positivity detected by immunohistochemistry (IHC) and FISH and the prognostic importance of MYC+ by IHC. When an optimal MYC immunostain is validated and cutoff standards established, we hope this will result in a hastening of the BL and BCL-U diagnoses [20] [21]. Starting with "less-intense chemotherapy" however, did not seem to negatively impact clinical outcomes (Figure 3), validating the importance of initiating early cytotoxic therapy in high-grade lymphomas, even if it is later changed. Indeed, the median time from diagnosis until the initiation of CHOP-R first was 50\% shorter compared with dmCODOX-M (9 vs 17d), which may be clinically important in a tumor with a proliferation rate of $90 \%-100 \%$. In addition, the use of R-CHOP upfront was associated with improved OS by univariate analysis. It is unknown if this apparent benefit is attributable to the use of rituximab, or the earlier initiation of chemotherapy before patients became sicker by allowing for some debulking of extensive disease [22] prior to more intensive dmCODOX-M.

B-cell lymphoma, unclassifiable, with features intermediate between diffuse large B-cell lymphoma and BL (BCL-U) is a challenging histology to treat with inferior outcomes to DLBCL when treated similarly [23] [24]. In this study, we demonstrate that the use of an aggressive regimen such as R-dmCODOX-M/IVAC can result in similar survival rates achievable in BL patients. With short follow-up, additional chemotherapy (such as RCHOP) following R-dmCODOX-M/IVAC appears promising for BCL-U patients. In support of this practice, Corazzelli et al. (2012) added two cycles of R-CODOX after R-dmCODOX-M/IVAC in such patients and demonstrated no significant difference in PFS between BL and BCL-U, and a BCL-U PFS rate higher than historical controls treated with standard CODOX-M/IVAC? [11].

Limitations of our study include the small sample size, the single center experience, and the variability of treatment regimens with respect to R use and timing. However, we feel this reflects "real world" BL/BCL-U treatment, where the diagnosis of true BL is imperfect and often delayed and the patient population is sicker and older than that of clinical trials.

In summary, dmCODOX-M/IVAC+/- $\mathrm{R}$ chemotherapy for older, high risk BL/BCL-U patients provided reasonable two-year OS and PFS. Rituximab significantly improved overall and progression free survival and should be incorporated into all future deployments of this regimen. While no differences in outcomes were observed between BL and BCL-U, the addition of extra therapy to BCL-U patients after the completion of RdmCODOX-M-IVAC is encouraging and needs further study.

\section{Clinical Practice Points}

Sporadic Burkitt lymphoma (BL) and B-cell lymphoma, unclassifiable (BCL-U), with features intermediate between diffuse large B-cell lymphoma (DLBCL) and BL are rare, highly aggressive CD 20+ B-cell non-Hodgkin's lymphomas characterized by a growth fraction approaching $100 \%$ and MYC translocations. BCL-U is a challenging histology to treat with inferior outcomes to DLBCL when treated similarly. In recent years, many adult cases of BL and BCL-U have been treated with a rapidly cycling, high intensity pediatric regimen of CODOX-M/IVAC. The addition of rituximab to CODOX-M/IVAC is relatively new in both BL and BCL-U. In our cohort of 31 patients, we found that rituximab significantly improved overall and progression-free survival. Unfortunate delays in diagnosis are common with this disease in "real-world practice" and 32 percent of our pa- 
tients were initially misclassified as DLBCL and treated with chemotherapy other than CODOX-M for cycle \# 1 (mostly R-CHOP). Starting with "less-intense chemotherapy" did not seem to negatively impact clinical outcomes, with no difference in PFS and OS between the two groups. With short follow-up, additional chemotherapy (such as R-CHOP) following R-dmCODOX-M/IVAC appears promising for BCL-U patients. Thus, Rituximab significantly improved overall and progression free survival and should be incorporated into all future deployments of this regimen. Starting with R-CHOP while awaiting confirmatory studies does not affect outcomes, validating the importance of initiating early cytotoxic therapy in high-grade lymphomas. While no differences in outcomes were observed between BL and BCL-U, the addition of extra therapy to BCL-U patients after the completion of R-dmCODOX-M-IVAC is encouraging and needs further study.

\section{Funding}

This work was in part supported by a Leukemia/Lymphoma Society of Canada summer studentship grant (KT).

\section{Contributions}

AP conducted chart review, oversaw data analysis and co-wrote the manuscript;

AP conducted chart review and helped write the manuscript;

MC contributed patients and helped write the manuscript;

KT conducted chart review;

GP contributed patients and helped edit the manuscript;

NB contributed patients and helped edit the manuscript;

KI contributed patients and helped edit the manuscript;

DG provided pathological review and helped edit the manuscript;

LZ provided statistical analysis and helped edit the manuscript;

$\mathrm{AV}$ and RP contributed therapy related information and helped edit the manuscript;

RB supervised the research, oversaw data synthesis and analysis and co-wrote the manuscript.

\section{Disclosure}

The authors have declared no conflicts of interest.

\section{References}

[1] Swerdlo, S., Campo, E., Harris, N., et al. (2008) WHO Classification of Tumours of Haematopoietic and Lymphoid Tissues. IARC Press, Lyon.

[2] Haralambieva, E., Boerma, E.J., Van Imhoff, G.W., et al. (2005) Clinical, Immunophenotypic, and Genetic Analysis of Adult Lymphomas with Morphologic Features of Burkitt Lymphoma. The American Journal of Surgical Pathology, 29, 1086-1094.

[3] Magrath, I., Adde, M., Shad, A., et al. (1996) Adults and Children with Small Non-Cleaved-Cell Lymphoma Have a Similar Excellent Outcome When Treated with the Same Chemotherapy Regimen. Journal of Clinical Oncology, 14, 925-934.

[4] Magrath, I.T., Janus, C. and Sariban, E. (1984) Therapy of Localized Non-Hodgkin’s Lymphoma in Children. Journal of Clinical Oncology, 2, 140-141.

[5] Mead, G.M., Barrans, S.L., Qian, W., et al. (2008) A Prospective Clinicopathologic Study of Dose-Modified CODOXM/IVAC in Patients with Sporadic Burkitt Lymphoma Defined Using Cytogenetic and Immunophenotypic Criteria (MRC/NCRI LY10 Trial). Blood, 112, 2248-2260. http://dx.doi.org/10.1182/blood-2008-03-145128

[6] Lacasce, A., Howard, O., Lib, S., et al. (2004) Modified Magrath Regimens for Adults with Burkitt and Burkitt-Like Lymphomas: Preserved Efficacy with Decreased Toxicity. Leukemia \& Lymphoma, 45, 761-767. http://dx.doi.org/10.1080/1042819031000141301

[7] Mead, G.M., Sydes, M.R., Walewski, J., et al. (2002) An International Evaluation of CODOX-M and CODOX-M Alternating with IVAC in Adult Burkitt's Lymphoma: Results of United Kingdom Lymphoma Group LY06 study. Annals of Oncology, 13, 1264-1274. http://dx.doi.org/10.1093/annonc/mdf253

[8] Barnes, J.A., Lacasce, A.S., Feng, Y., et al. (2011) Evaluation of the Addition of Rituximab to CODOX-M/IVAC for Burkitt's Lymphoma: A Retrospective Analysis. Annals of Oncology, 22, 1859-1864.

http://dx.doi.org/10.1093/annonc/mdq677 
[9] Maruyama, D., Watanabe, T., Maeshima, A.M., et al. (2010) Modified Cyclophosphamide, Vincristine, Doxorubicin, and Methotrexate (CODOX-M)/Ifosfamide, Etoposide, and Cytarabine (IVAC) Therapy with or without Rituximab in Japanese Adult Patients with Burkitt Lymphoma (BL) and B Cell Lymphoma, Unclassifiable, with Features Intermediate between Diffuse Large B Cell Lymphoma and BL. International Journal of Hematology, 92, 732-743. http://dx.doi.org/10.1007/s12185-010-0728-0

[10] Mohamedbhai, S.G., Sibson, K., Marafioti, T., et al. (2011) Rituximab in Combination with CODOX-M/IVAC: A Retrospective Analysis of 23 Cases of Non-HIV Related B-Cell Non-Hodgkin Lymphoma with Proliferation Index >95\%. British Journal of Haematology, 152, 175-181. http://dx.doi.org/10.1111/j.1365-2141.2010.08447.x

[11] Corazzelli, G., Frigeri, F., Russo, F., Frairia, C., Arcamone, M., Esposito, G., et al. (2012) RD-CODOX-M/IVAC with Rituximab and Intrathecal Liposomal Cytarabine in Adult Burkitt Lymphoma and "Unclassifiable” Highly Aggressive B-Cell Lymphoma. British Journal of Haematology, 156, 234-244. http://dx.doi.org/10.1111/j.1365-2141.2011.08947.x

[12] Jaffe, E., Harris, N.L., Stein, H. and Vardiman, J., Eds. (2001) Tumours of Haematopoietic and Lymphoid Tumours. AIARC Press, Lyon, 181-184.

[13] Cheson, B.D., Horning, S.J., Coiffier, B., Shipp, M.A., Fisher, R.I., Connors, J.M., et al. (1999) Report of an International Workshop to Standardize Response Criteria for Non-Hodgkin's lymphomas. NCI Sponsored International Working Group. Journal of Clinical Oncology: Official Journal of the American Society of Clinical Oncology, 17, 1244.

[14] Bernstein, J.I., Coleman, C.N., Strickler, J.G., Dorfman, R.F. and Rosenberg, S.A. (1986) Combined Modality Therapy for Adults with Small Noncleaved Cell Lymphoma (Burkitt's and Non-Burkitt's Types). Journal of Clinical Oncology: Official Journal of the American Society of Clinical Oncology, 4, 847-858.

[15] Abramson, J.S., Barnes, J.A., Toomey, C.E., et al. (2008) Rituximab Added to CODOX-M/IVAC Is Highly Effective in HIV-Negative and HIV-Positive Burkitt Lymphoma. ASH Annual Meeting Abstracts, 112, 3595.

[16] Blum, K.A., Lozanski, G. and Byrd, J.C. (2004) Adult Burkitt Leukemia and Lymphoma. Blood, 104, 3009-3020. http://dx.doi.org/10.1182/blood-2004-02-0405

[17] Wästerlid, T., Brown, P.N., Hagberg, O., Hagberg, H., Pedersen, L.M., D’Amore, F. and Jerkeman, M. (2013) Impact of Chemotherapy Regimen and Rituximab in Adult Burkitt Lymphoma: A Retrospective Population-Based Study from the Nordic Lymphoma Group. Annals of Oncology, 24, 1879-1886. http://dx.doi.org/10.1093/annonc/mdt058

[18] Barnes, J.A., Lacasce, A.S., Feng, Y., Toomey, C.E., Neuberg, D., Michaelson, J.S., Hochberg, E.P. and Abramson, J.S. (2011) Evaluation of the Addition of Rituximab to CODOX-M/IVAC for Burkitt's Lymphoma: A Retrospective Analysis. Annals of Oncology, 22, 1859-1864. http://dx.doi.org/10.1093/annonc/mdq677

[19] Ribrag, V., Koscielny, S., Bouabdallah, K., Herbrecht, R., Iacobelli, S., Bosq, J., et al. (2012) Addition of Rituximab Improves Outcome of HIV Negative Patients with Burkitt Lymphoma Treated with the Lmba Protocol: Results of the Randomized Intergroup (GRAALL-Lysa) LMBA02 Protocol. (IGR Sponsored LMBA02, NCT00180882). ASH Annual Meeting Abstracts, 120, 685.

[20] Green, T.M., Young, K.H., Visco, C., Xu-Monette, Z.Y., Orazi, A., Go, R.S., et al. (2012) Immunohistochemical Double-Hit Score Is a Strong Predictor of Outcome in Patients with Diffuse Large B-Cell Lymphoma Treated with Rituximab Plus Cyclophosphamide, Doxorubicin, Vincristine, and Prednisone. Journal of Clinical Oncology, 30, 34603467. http://dx.doi.org/10.1200/JCO.2011.41.4342

[21] Johnson, N.A., Slack, G.W., Savage, K.J., Connors, J.M., Ben-Neriah, S., Rogic, S., et al. (2012) Concurrent Expression of MYC and BCL2 in Diffuse Large B-Cell Lymphoma Treated with Rituximab Plus Cyclophosphamide, Doxorubicin, Vincristine, and Prednisone. Journal of Clinical Oncology, 30, 3452-3459. http://dx.doi.org/10.1200/JCO.2011.41.0985

[22] Pfreundschuh, M., Trümper, L., Kloess, M., Schmits, R., Feller, A.C., Rübe, C., et al. (2004) Two-Weekly or 3-Weekly CHOP Chemotherapy with or without Etoposide for the Treatment of Elderly Patients with Aggressive Lymphomas: Results of the NHL-B2 Trial of the DSHNHL. Blood, 104, 634-641. http://dx.doi.org/10.1182/blood-2003-06-2095

[23] Smeland, S., Blystad, A.K., Kvaløy, S.O., Ikonomou, I.M., Delabie, J., Kvalheim, G., Hammerstrøm, J., Lauritzsen, G.F. and Holte, H. (2004) Treatment of Burkitt's/Burkitt-Like Lymphoma in Adolescents and Adults: A 20-Year Experience from the Norwegian Radium Hospital with the Use of Three Successive Regimens. Annals of Oncology, 15, 1072-1078. http://dx.doi.org/10.1093/annonc/mdh262

[24] Perry, A.M., Crockett, D., Dave, B.J., Althof, P., Winkler, L., Smith, L.M., et al. (2013) B-Cell Lymphoma, Unclassifiable, with Features Intermediate between Diffuse Large B-Cell Lymphoma and Burkitt Lymphoma: Study of 39 Cases. British Journal of Haematology, 162, 40-49. http://dx.doi.org/10.1111/bjh.12343 
Scientific Research Publishing (SCIRP) is one of the largest Open Access journal publishers. It is currently publishing more than 200 open access, online, peer-reviewed journals covering a wide range of academic disciplines. SCIRP serves the worldwide academic communities and contributes to the progress and application of science with its publication.

Other selected journals from SCIRP are listed as below. Submit your manuscript to us via either submit@scirp.org or Online Submission Portal.
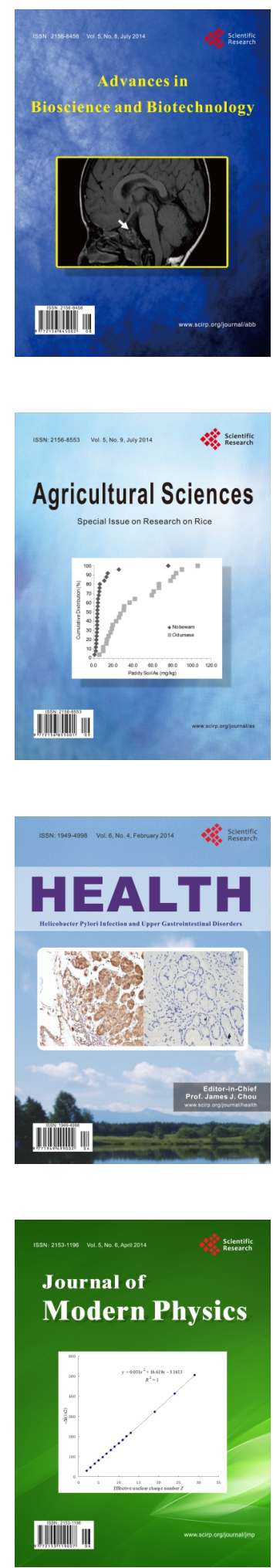
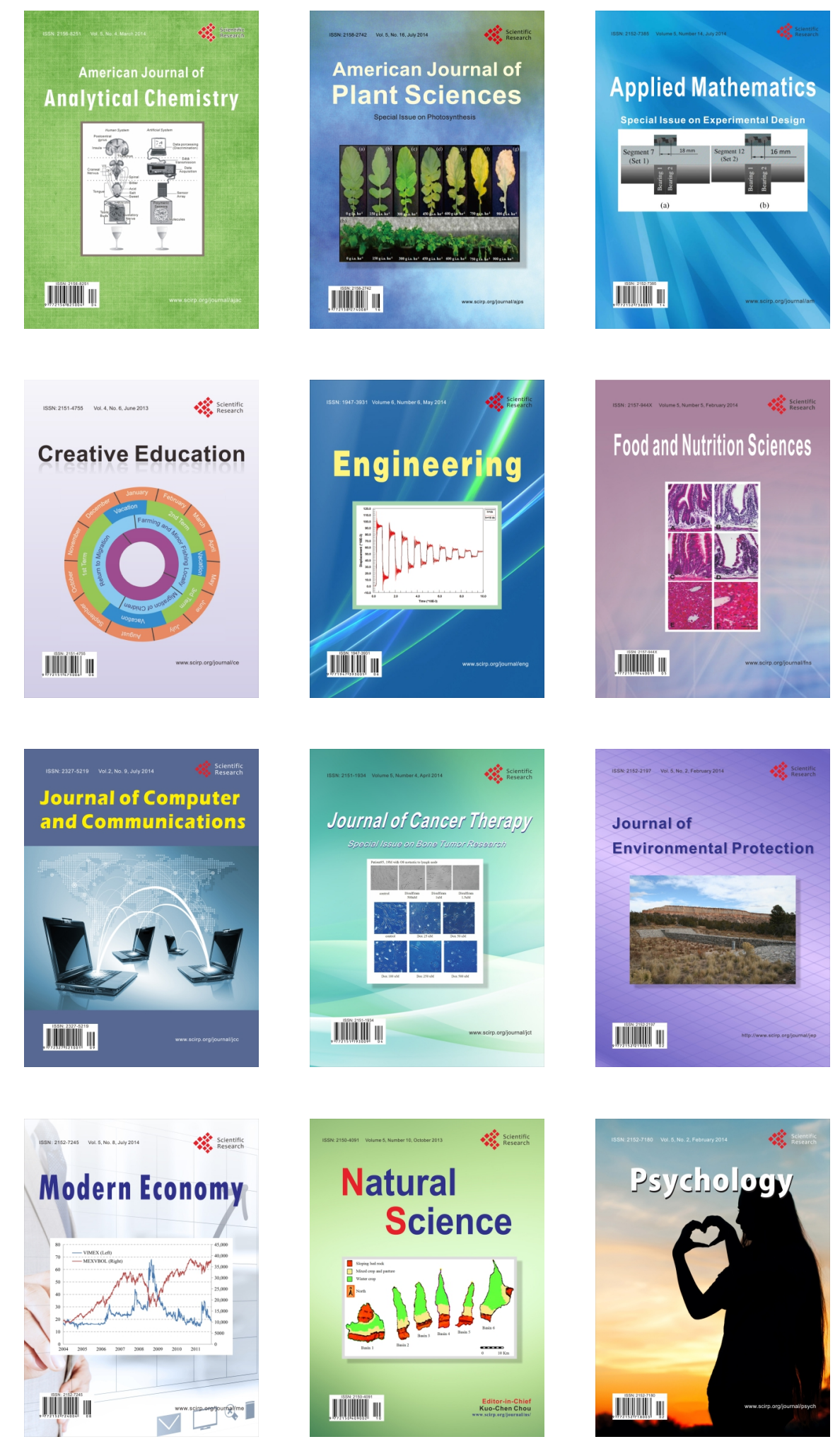